10. Mr. L. B. Robinson: A generalization of the notion of covariants.

Riquier has generalized the theory of complete systems. Mr. Robinson shows that a generalized complete system

$$
\frac{\partial w_{i}}{\partial x_{j}}=\sum_{r=1}^{n} A_{r j} \frac{\partial w_{i}}{\partial t_{r}}+U_{i j}
$$

can be utilized to generalize the notion of covariants. The generalized covariants are solutions of the above system and can be obtained by a finite number of differentiations or integrations.

11. Dr. G. A. Campbell: Inductances of grounded circuits.

Any network of conductors located on the surface of the earth with which it is conductively connected at any number of points will, for direct currents, have self and mutual inductances which are equal to the Neumann integral extended over the network alone. In other words, that portion of the complete Neumann integral for closed circuits which involves the return currents through the earth vanishes. The earth is assumed to be flat, of infinite extent, of unit permeability and of uniform conductivity.

R. G. D. Richardson, Secretary.

\title{
THE OCTOBER MEETING OF THE SAN FRANCISCO SECTION
}

The thirty-eighth regular meeting of the San Francisco Section was held at the University of California on Saturday, October 22, 1921. Professor Lehmer presided at the earlier part of the meeting, later relieved by Professor Allardice. The total attendance was twenty-five, including the following seventeen members of the Society:

Alderton, Allardice, Bernstein, Blichfeldt, Buck, Cajori, Daus, Edwards, Haskell, Hoskins, Lehmer, Moreno, F. R. Morris, Noble, T. M. Putnam, Pauline Sperry, A. R. Williams.

The following officers were elected for the year: Chairman, Professor Allardice; Secretary, Professor Bernstein; programme committee, Professors Blichfeldt, Lehmer, Bernstein. 
The dates of the next two meetings were fixed as April 8, 1922, and October 21, 1922.

Titles and abstracts of the papers read at this meeting follow.

Mr. Wong was introduced by Professor Lehmer. In the absence of the authors, the papers of Professor Moritz and Professor Bell were read by title.

1. Professor Thomas Buck: A class of asymptotic solutions of the restricted problem of three bodies. Preliminary report.

The solutions in question are of the type considered by Poincaré, and are asymptotic to the Lagrangian equilateral triangle solutions.

2. Professor Florian Cajori: Spanish and Portuguese symbols for "thousands."

The Spanish symbol named calderón, designating "thousand," found in Spanish and Spanish-American manuscripts as well as in printed books as far back as the fifteenth century, is identified by Professor Cajori as being the same as a symbol for "thousand" used in northwestern Italy during the fifteenth century. The calderón, the author points out, is probably a modified form of the Roman symbol CID for "thousand." The Portuguese cifrão resembles our \$, and is used like the Spanish calderón, having probably the same origin.

3. Professor Florian Cajori: History of notations of the calculus.

The history of early calculus notations is given in detail in our histories of mathematics and books relating to Newton and Leibniz. Cantor's Geschichte der Mathematik, vol. 4, for the years 1759-1799, pays little attention to calculus notations of that period, and no detailed history of the nineteenth century notations exists. The present paper endeavors to cover the period since about 1740 .

4. Mr. B. C. Wong: A theorem on double points.

The following theorem is proved: The total number $D$ of double points on a composite curve of order $n$ made up of $r$ curves is greater by $r-1$ than the maximum number $D$ of double points on a proper curve of the same order diminished by the sum $P$ of the deficiencies of the $r$ curves; that is $D=D^{\prime}+r-1-P$. 
5. Professor B. A. Bernstein: On complete independence of Hurwitz's postulates for abelian groups and fields.

Professor Bernstein discusses the question of complete independence of each of the postulate-sets for abelian groups and fields presented by Hurwitz in the AnNals of MatheMATICS, (2), vol. 15 (1913), p. 93.

6. Dr. A. R. Williams: On finding the rate of interest on a given investment.

The writer shows that in finding the rate of interest yielded by a redeemable security it is generally advantageous to reduce the equation to the form $i=f(i)$, where $f(i)$ is a rational function of $i$, whose rate of change with respect to $i$ can be calculated if desired. It is then easy to find by trial a value of $i$ to make the two members agree to any desired number of places. The method is self-checking, and is particularly easy of application when $f(i)$ is a decreasing function, as will be the case when the redemption price is greater than the purchase price.

7. Professor R. E. Moritz: Anti-ratientiation; definition and fundamental relations.

In a paper published in the American Journal, vol. 24, pp. 257-302, Professor Moritz considered certain general limiting processes of the $n$th order (ratientiation) which include the differentiation and quotientiation processes as special cases corresponding to $n=0$ and $n=1$ respectively. In the present paper the corresponding inverse processes are defined and their fundamental relations developed. The results will be embodied in a more comprehensive paper which will appear in the TôHокU JournaL.

8. Professor E. T. Bell: Extensions of Dirichlet multiplication and Dedekind inversion.

In this paper, which appears in this number of the BULLETIN, the author develops the theory of fields in which multiplication and division are extensions of processes defined by Cauchy, Dirichlet and Dedekind for series. The theory has been devised for the specific purpose of simplifying and correlating much of the material summarized in volume $I$, chapters V, X, XIX of Dickson's History of the Theory of Numbers. 
9. Professor E. T. Bell: A qualitative theory of singly infinite series and products.

The object of the theory in Professor Bell's second paper is to continue that of his first and to determine under what conditions, useful in the theory of numbers, infinite series and infinite products can be manipulated to yield arithmetic theorems without any reference to their convergence or divergence.

Three kinds of multiplication of series and their inverses are discussed in detail. The third, Euler multiplication and division, is new, and connects the Dirichlet and Cauchy multiplication of series with the expansion of products of an infinite number of infinite series. Complete algebras of these processes are constructed, and each is shown to be abstractly identical with common algebra.

10. Professor E. T. Bell: Arithmetical equivalents for a remarkable relation between theta functions.

In a former paper, to appear shortly in the GIORNaLE DI Matematiche, Professor Bell gave a complete system of arithmetic equivalents for the equation of three terms in elliptic functions.

The present paper gives equivalents of a remarkable relation, due to Hermite and Enneper, which is complementary to the equation of three terms.

11. Professor E. T. Bell: The arithmetic of the nodes and tropes on a Kummer surface.

In a previous paper, to appear in the American Journal, Professor Bell remarked that the configuration of nodes and tropes on the Kummer surface is formally equivalent, in the sense of mathematical logic, to a system of theorems on the simultaneous representation of a pair of integers each as a sum of four squares.

This paper presents the equivalence which, for lack of space, was omitted from the other.

B. A. Bernstein, Secretary of the Section. 